\title{
Preservation vs Extraction of Carious Multirooted Teeth
}

\author{
Resmije Ademi-Abdyli ${ }^{1}$, Feriall Perjuci ${ }^{2}$, Yll Abdyli ${ }^{3}$, Teuta Bicaj ${ }^{4}$
}

\begin{abstract}
Introduction: Hemisection refers to the surgical separation of multirooted teeth when the decay or resorption is restricted to one root, followed by removal of the affected root with preservation of the healthy root with its crown portion and its usage as abutment for subsequent prosthetic restoration.

Aim: The aim of this case report was to raise awareness among dentists that preservation of tooth structure in the form of hemisection may be considered an alternative treatment modality in cases of multirooted teeth with extensive caries restricted to one of the roots.

Case description: This describes the hemisection procedure of right mandibular second molar performed in a 35-year-old female referred for extraction. Intraoral examination and intraoral periapical (IOPA) radiography revealed extensive decay of tooth 47 , restricted to the distal root, with satisfactory condition of the mesial root and its crown portion to be preserved by hemisection and used as abutment for a fixed prosthetic restoration. 6 weeks after the successful hemisection, a three-unit metal-porcelain fixed denture was performed, using the retained mesial root of mandibular second molar (47) and the mandibular second premolar (45) as abutments and replacing the missing mandibular first molar (46). Conclusion: Preservation of tooth structure by performing hemisection in carious multirooted teeth in cases when caries is restricted to one of the roots should be considered a valuable alternative treatment instead of extraction; therefore, it should be discussed with patients as one of the treatment options whenever suitable and possible to perform.

Clinical significance: The rationale of hemisection in our case is that it enabled the preservation of tooth structure by supplying an abutment for construction of a three-unit fixed partial denture, thus providing the patient the opportunity to maintain occlusal function with reduced financial cost over dental implants.

Keywords: Abutment, Decay, Fixed denture, Hemisection, Mandibular molar, Multirooted, Preservation.

World Journal of Dentistry (2019): 10.5005/jp-journals-10015-1683
\end{abstract}

\section{INTRODUCTION}

Loss of posterior teeth is an undesirable and concerning event, often leading to drifting of teeth, loss of masticatory function, and loss of arch length, which requires preventive and maintenance measures. ${ }^{1}$

Recent advances in dentistry and increased patient awareness regarding the importance of natural dentition and oral health in general have enabled the retention of carious multirooted teeth, refraining from their extraction and thereby permitting a longer lifespan of the natural dentition and its related functions. ${ }^{1,2}$

Various surgical procedures, such as hemisection, bisection, and trisection, are utilized to salvage multirooted teeth presenting with extensive caries. ${ }^{3}$

The procedure of hemisection refers to the surgical separation of a multirooted tooth, principally molars of the lower jaw succeeded by extraction of the affected part of the tooth.

In cases where the carious lesion, potential vertical root fracture, or iatrogenic root perforation is contained within one of the roots as well as in cases of furcation involvement, hemisection can be considered as a viable treatment modality, eschewing tooth extraction and sparing the healthy root, enabling it to act as abutment for the subsequent fixed prosthetic restoration. ${ }^{4-6}$

The residual root can be restored either individually by full crown coverage or included as abutment of a fixed partial denture/ bridge. $^{7}$

\section{Case Description}

A 35-year-old female patient attended the Oral Surgery Department of the University Dental Clinical Centre of Kosovo for extraction of tooth 47 with recommendation of her prosthodontist. Intraoral clinical examination revealed extensive decay under the old
1,2Department of Oral Surgery, University Dental Clinical Centre, Dentistry School, Medical Faculty, University of Prishtina, Prishtina, Kosovo

${ }^{3}$ Dentistry School, Medical Faculty, University of Prishtina, Prishtina, Kosovo

${ }^{4}$ Department of Prosthodontics, University Dental Clinical Centre, Dentistry School, Medical Faculty, University of Prishtina, Prishtina, Kosovo

Corresponding Author: YII Abdyli, Dentistry School, Medical Faculty, University of Prishtina, Prishtina, Kosovo, Phone: +383 44131414, e-mail:yllabdyli10@gmail.com

How to cite this article: Ademi-Abdyli R, Perjuci F, Abdyli $Y$, et al. Preservation vs Extraction of Carious Multirooted Teeth. World J Dent 2019;10(6):466-469.

Source of support: Nil

Conflict of interest: None

composite restorations of teeth 47 and 45 , with the tooth 46 missing (Fig. 1).

Intraoral periapical (IOPA) radiograph revealed deep caries of the distal half of the tooth 47, involving the distal root and causing its complete separation from the crown portion. Radiography also revealed excellent periodontal support of mesial root of tooth 47, although the endodontic root canal treatment seemed unsatisfactory (Fig. 2A). According to the prosthodontist, the potential therapy for this case was extraction of tooth 47 and replacement of missing teeth 46 and 47 with dental implants or removable partial denture.

Considering that the patient ruled out dental implants due to insufficient funds and the fact that she did not want to wear 
removable dentures because of her young age, we offered the option of hemisection of the distal root of tooth 47 and replacement of the missing tooth 46 with a three-unit fixed partial denture

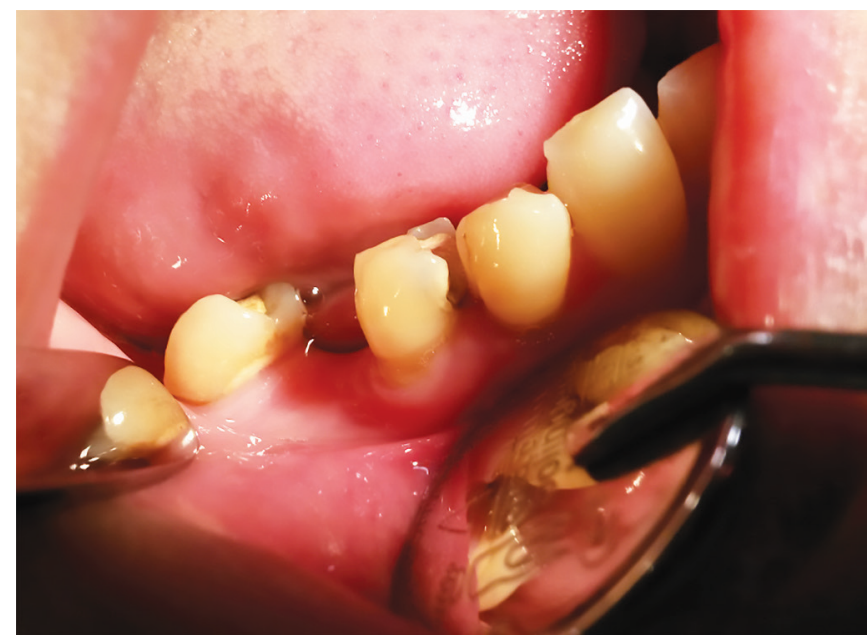

Fig. 1: Intraoral examination revealing carious lesions on teeth 45,47 , and missing tooth 46

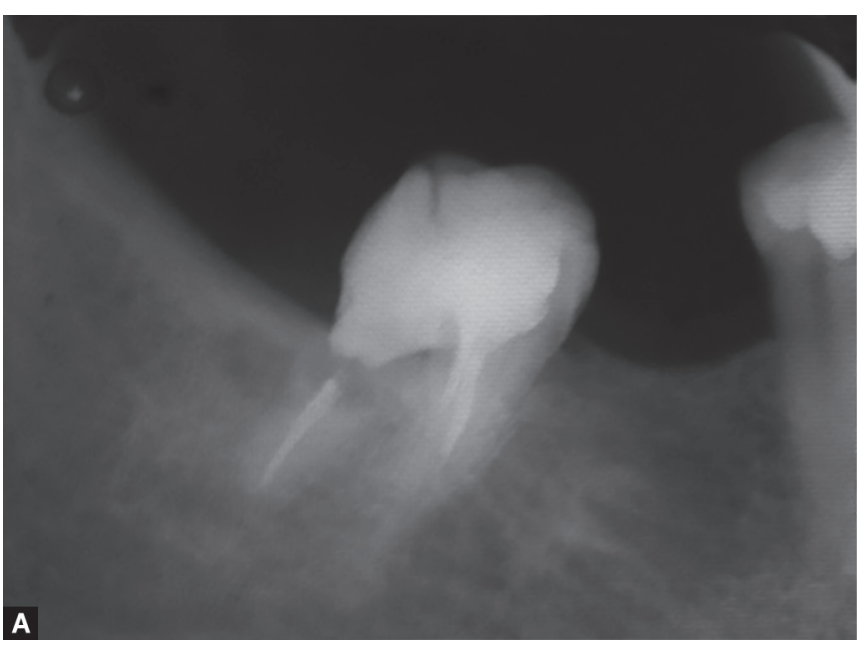

using teeth 45 and 47 as abutments. Hemisection was a suitable alternative to extraction because caries on tooth 47 was restricted to the distal root, while the mesial root and its surrounding bone were in satisfactory condition and the remaining crown portion was sufficient to act as an abutment.

Despite the unsatisfactory endodontic treatment of the mesial root canals, we did not suggest endodontic retreatment fearing the risk of root perforation during the procedure. The patient agreed to this option and the procedure of distal root hemisection of tooth 47 was performed under local anesthesia, using a long tapered fissure bur with sterile saline external cooling. Successful hemisection was confirmed by IOPA radiograph showing the retained mesial root of tooth 47 (Fig. 2B).

Treatment was completed after 6 weeks, a sufficient time for wound healing and periodontal stabilization of the remaining mesial root. The crown of tooth 45 and coronal part of the remaining root of tooth 47, used as abutments, were treated with composite materials and prepared with premolar morphology (Fig. 3A). After taking the impression using standard techniques, a three-unit metal framework was constructed and tried in the patient's mouth (Fig. 3B).

Figs 2A and B: (A) Initial intraoral periapical (IOPA) radiograph of tooth 47 reveals deep caries involving distal root, while mesial root shows excellent periodontal support but unsatisfactory endodontic treatment; (B) The IOPA radiographic condition of the retained mesial root of tooth 47 after successful hemisection
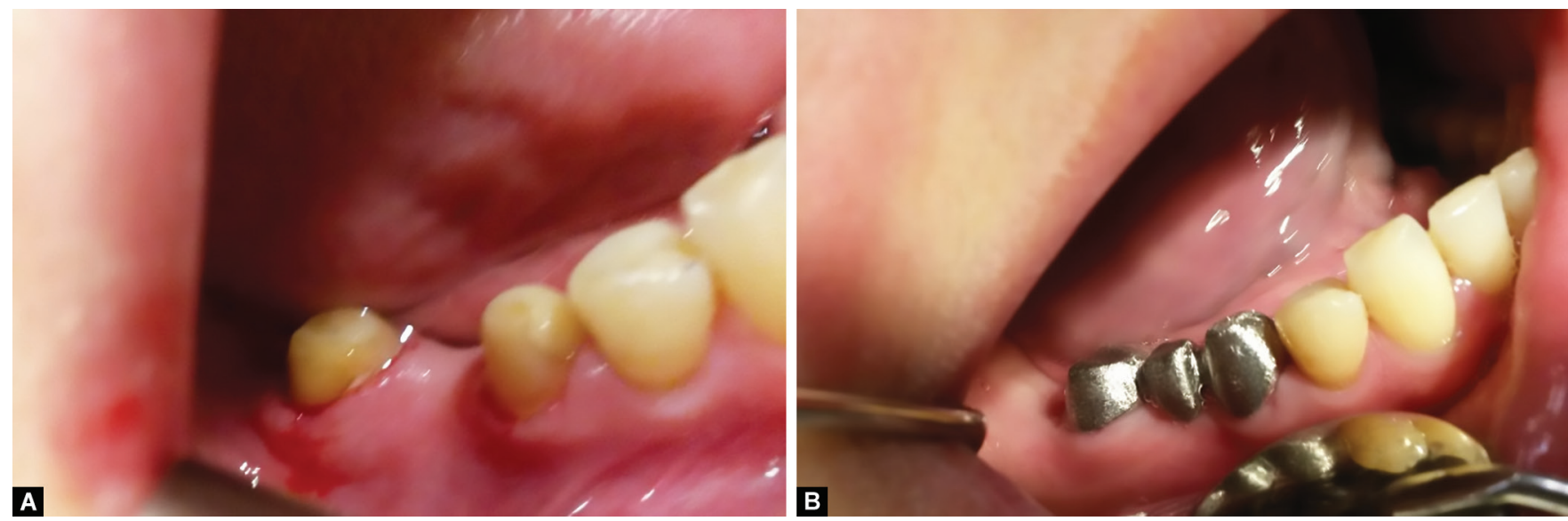

Figs $3 \mathrm{~A}$ and B: Intraoral condition 6 weeks after hemisection, during the course of treatment. (A) Carious tooth 45 and retained root of tooth 47 treated with resin-composite material and prepared for impression with premolar morphology; (B) Metal framework using tooth 45 and retained mesial root of tooth 47 as abutments 


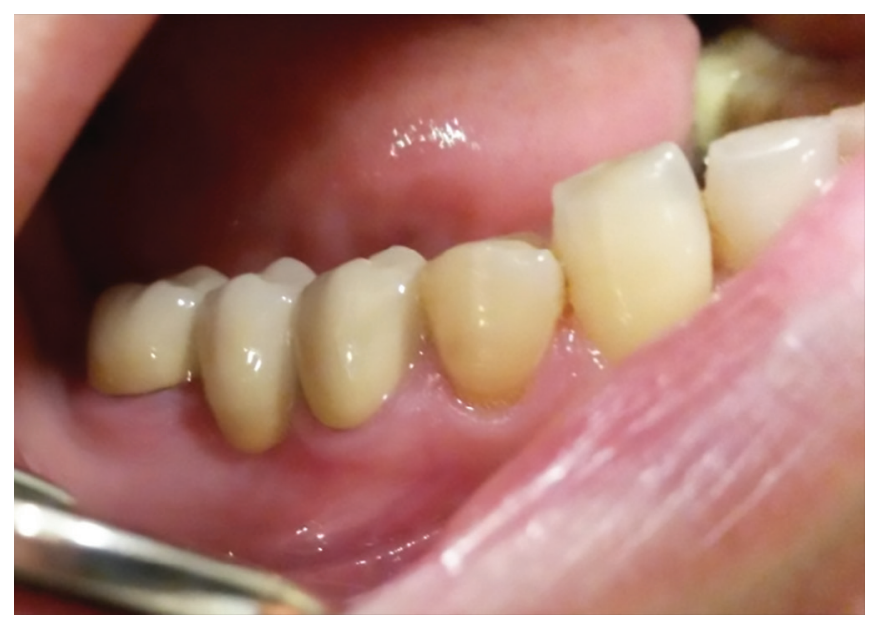

Fig. 4: Cemented fixed three-unit metal-porcelain restoration

Finally, the three-unit metal-porcelain restoration, consisting of teeth crowns 45,46 , and 47 , was completed and cemented using glass ionomer cement (Fig. 4).

Intraoral periapical radiograph confirmed excellent osculation of the prosthetic restoration with abutments. With the purpose of proper fitting of the metal-porcelain crown with the abutment, the vertical margin of the metal framework on the distal surface of the retained root of 47 was made longer, i.e., about $2-3 \mathrm{~mm}$ below the gingival margin compared with the mesial surface, due to the hard tissue resorption and soft tissues depression on the site of hemisection after the wound healing (Fig. 5).

\section{Discussion}

Management of periodontally involved molars with extensive caries and unrestorable condition is a challenging enterprise and is limited to dental extraction. ${ }^{8}$

If caries of multirooted teeth of lower jaw is limited to one root, and the other root is unharmed and robust, hemisection may be a suitable alternative to extraction. Hemisection is also indicated in cases, such as severe vertical bone loss around one root of a multirooted tooth, furcation destruction, nonrestorable portion of a multirooted tooth, bur perforations during endodontic access preparation or during search for canal orifices, and misdirected files during canal negotiation. ${ }^{9-11}$

In cases selected for hemisection in order to salvage a compromised multirooted tooth, root morphology should be taken into account, namely the root shape, length, curvature, and divergence, as well as the quality of the root canal treatment.

Presence of long and straight roots as well as divergent roots on teeth subjected to hemisection facilitates and favors the outcome of the hemisection procedure, whereas conjoined or proximate roots with inadequate amount of remaining alveolar bone to support the remaining root structure should not receive hemisection therapy. ${ }^{5,6}$

This presented case report illustrates resection of the distal root of mandibular molar, although literature shows that, more often, the distal root is retained with the mesial one being removed due to its anatomical structure.

Considering its broad and straight morphology, the distal root of mandibular molars is more appropriate to act as abutment, whereas the longitudinal groove of the mesial root reduces internal surface area, making it unsuitable for post and core restoration. ${ }^{12}$

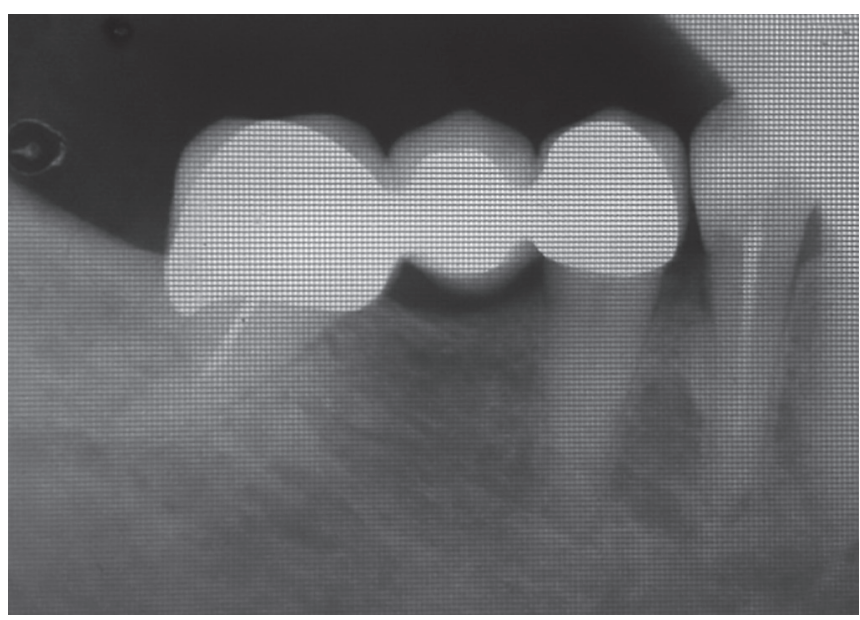

Fig. 5: Intraoral periapical radiograph demonstrating excellent osculation and proper margin fit of fixed metal-porcelain restoration, achieved with a longer vertical margin $(2-3 \mathrm{~mm})$ below the gingival margin on the distal surface of the metal-porcelain crown of the retained root tooth 47

The long-term therapeutic success of the hemisection procedure is dependent upon careful case selection and proper cooperation between dental specialists, namely oral surgeon, endodontist, and prosthodontist. ${ }^{6,13}$

Different treatment modalities can be considered in these situations, such as removable dentures, fixed partial dentures, or replacement of the missing tooth with dental implant.

In our reported case, despite the recommendation for extraction from the private prosthodontist, our decision as oral surgeons was to preserve the tooth by applying hemisection in consultation with the institution's prosthodontist as part of a multidisciplinary approach. After the successfully performed hemisection, maintenance of function and stabilization of occlusion using the hemisected tooth were achieved with a full-coverage three-unit fixed denture $(45,46$, and 47$)$, replacing the missing tooth 46 using tooth 45 and retained mesial root of tooth 47 as abutments.

Therefore, the hemisection procedure is a valuable and rational treatment modality, especially in young patients, as it enables preservation of tooth structure, which, by acting as abutment for construction of fixed partial denture, provides the patient with the possibility of maintaining function and stabilization of occlusion with reduced psychological trauma and financial cost compared with other treatment options, such as dental implants.

\section{Conclusion}

Preservation of tooth structure by performing hemisection in carious multirooted teeth in cases where caries is restricted to one of the roots should be considered a valuable alternative treatment instead of extraction; therefore, it should be discussed with patients as one of the treatment options, whenever suitable and possible to perform.

\section{Clinical Significance}

The rationale of hemisection in our case is that it enabled preservation of tooth structure by supplying an abutment for construction of a three-unit fixed partial denture, thus providing the patient the opportunity to maintain occlusal function with reduced financial cost over dental implants. 


\section{References}

1. Jain A, Bahuguna R, Agarwal V. Hemisection as an alternative treatment for resorbed multirooted tooth - a case report. Asian $J$ Oral Health Allied Sci 2011;1(1):44-46.

2. Mittal P, Prasad AB, Raisingai $D$, et al. hemisection: a savior for hopeless teeth. J Mahatma Gandhi Univ Med Sci Tech 2016;1(1):30-34. DOI: 10.5005/jp-journals-10057-0008.

3. Rajesh H, Dharamasi A, Rajesh KS, et al. Root resection: apropos of 6 cases. Arch Med Health Sci 2014;2(2):209-213. DOI: 10.4103/23214848.144342.

4. Kost WJ, Stakiw JE. Root amputation and hemisection. J Can Dent Assoc 1991;57(1):42-45.

5. Parmar G, Vashi P. Hemisection: a case-report and review. Endodontology 2003;15:26-29.

6. Kurtzman GM, Mahesh L, Qureshi I. Hemisection as an alternative treatment for the vertically fractured mandibular molar. J Pak Dent Assoc 2012;21(3):177-181.
7. Naveen YG, Patel JR, Parikh P, et al. Alternatives for restoration of a hemisected mandibular molar. BMJ Case Rep 2014;2014:010275. DOI: 10.1136/bcr-2013-010275.

8. Radke $U$, Kubde R, Paldiwal A. Hemisection: A window of hope for freezing tooth. Case Rep Dent 2012;2012:390874. DOI: $10.1155 / 2012 / 390874$.

9. Regan JD, Witherspoon DE, Foyle D. Surgical repair of root and tooth perforations. Endod Topics 2005;11(1):152-178. DOI: 10.1111/j.16011546.2005.00183.x.

10. Shah S, Modi B, Desai K, et al. Hemisection-a conservative approach for a periodontally compromised tooth-a case report. J Adv Oral Res 2012;3(2):31-35. DOI: 10.1177/2229411220120205.

11. Menezes R, da Silva Neto UX, Carneiro E, et al. MTA repair of a supracrestal perforation: a case report. J Endod 2005;31(3):212-214. DOI: 10.1097/01.don.0000137639.85637.67.

12. Vikram M, Vivekananda Pai AR. Salvaging a tooth by hemisection. Health Renaiss 2012;10(3):251-253. DOI: 10.3126/hren.v10i3.7057.

13. Akki S, Mahoorkar S. Tooth hemisection and restoration an alternative to extraction - a case report. Int J Dent Clin 2011;3(3):67-68. 